\title{
Quantitative Determination of Temperature in the Approach to Magnetic Order of Ultracold Fermions in an Optical Lattice
}

\author{
R. Jördens, ${ }^{1}$ L. Tarruell, ${ }^{1}$ D. Greif, ${ }^{1}$ T. Uehlinger, ${ }^{1}$ N. Strohmaier, ${ }^{1}$ H. Moritz, ${ }^{1}$ T. Esslinger, ${ }^{1, *}$ L. De Leo, ${ }^{2}$ C. Kollath, ${ }^{2}$ \\ A. Georges,${ }^{2,3}$ V. Scarola, ${ }^{4}$ L. Pollet,${ }^{5}$ E. Burovski, ${ }^{6}$ E. Kozik, ${ }^{1}$ and M. Troyer ${ }^{1}$ \\ ${ }^{1}$ Department of Physics, ETH Zurich, 8093 Zurich, Switzerland \\ ${ }^{2}$ Centre de Physique Théorique, CNRS, École Polytechnique, 91128 Palaiseau Cedex, France \\ ${ }^{3}$ Collège de France, 11 place Marcelin Berthelot, 75231 Paris Cedex, France \\ ${ }^{4}$ Department of Physics, Virginia Tech, Blacksburg, Virginia 24061, USA \\ ${ }^{5}$ Department of Physics, Harvard University, Cambridge, Massachusetts 02138, USA \\ ${ }^{6}$ LPTMS, CNRS and Université Paris-Sud, UMR8626, Bâtiment 100, 91405 Orsay, France \\ (Received 18 December 2009; revised manuscript received 23 March 2010; published 6 May 2010)
}

\begin{abstract}
We perform a quantitative simulation of the repulsive Fermi-Hubbard model using an ultracold gas trapped in an optical lattice. The entropy of the system is determined by comparing accurate measurements of the equilibrium double occupancy with theoretical calculations over a wide range of parameters. We demonstrate the applicability of both high-temperature series and dynamical mean-field theory to obtain quantitative agreement with the experimental data. The reliability of the entropy determination is confirmed by a comprehensive analysis of all systematic errors. In the center of the Mott insulating cloud we obtain an entropy per atom as low as $0.77 k_{B}$ which is about twice as large as the entropy at the Néel transition. The corresponding temperature depends on the atom number and for small fillings reaches values on the order of the tunneling energy.
\end{abstract}

DOI: 10.1103/PhysRevLett.104.180401

PACS numbers: 05.30.Fk, 03.75.Ss, 67.85.-d, 71.10.Fd

Experimental progress in the field of atomic quantum gases has led to a new approach to quantum many-body physics. In particular, the combination of quantum degenerate and strongly interacting Fermi gases [1,2] with optically induced lattice potentials [3] now allows the study of a centerpiece of quantum condensed matter physics, the Fermi-Hubbard model [4]. The high level of control over the atomic systems has led to the concept of quantum simulation, which for the case of the Fermi-Hubbard model is expected to provide answers to intriguing open questions of frustrated magnetism and $d$-wave superfluidity [5]. Recent experiments [6,7] have indeed demonstrated that the strongly correlated regime of the repulsive FermiHubbard model is experimentally accessible and the emergence of a Mott insulating state has been observed. In this Letter, we succeed in performing a quantitative simulation of the Fermi-Hubbard model using cold atoms. The level of precision of the experiment enables us to determine the entropy and the temperature of the system, and thereby to quantify the approach to the low temperature phases.

The main challenge for the quantum simulation of the Fermi-Hubbard model is a further reduction in temperature. Here the lack of a quantitative thermometry method in the lattice is a key obstacle. For strongly correlated bosonic systems thermometry has recently been demonstrated by direct comparison with quantum Monte Carlo simulations [8] or by using the boundary of two spin polarized clouds [9]. In the fermionic case, previous methods to determine the temperature could be used only in limiting regimes of the Hubbard model, namely, the noninteracting $[10,11]$ and zero-tunneling $[6,12]$ regimes. However, intermediate interactions are most interesting for quantum simulation of the Fermi-Hubbard model and no reliable thermometry method has been available up to now.

In both the metallic and Mott insulating regimes an accurate measurement of the double occupancy provides direct access to thermal excitations. We analyze the crossover from thermal creation of double occupancies to thermal depletion which is unique to a trapped system (see Fig. 1). The variability of the double occupancy with respect to temperature allows the entropy of the system to be inferred directly by comparison with two ab initio theoretical methods. By determining all other quantities entering the analysis separately and with methods that are independent of the double occupancy measurement, we demonstrate the versatility of this probe for quantifying the state of the system.

To obtain a quantum degenerate Fermi gas we adhere to the procedure described in previous work [6]. A balanced spin mixture of ${ }^{40} \mathrm{~K}$ atoms in the $m_{F}=-9 / 2$ and $-5 / 2$ magnetic sublevels of the $F=9 / 2$ hyperfine manifold is evaporatively cooled in a crossed beam optical dipole trap, with less than $1.2 \%$ of the atoms remaining in the $m_{F}=$ $-7 / 2$ state. We prepare Fermi gases with total atom numbers between $N=30 \times 10^{3}$ and $300 \times 10^{3}$. The atom number is calibrated using strong saturation imaging [13] at high magnetic field, with a systematic error $\sim 10 \%$.

The optical lattice potential is then ramped up in $0.2 \mathrm{~s}$ and has a simple cubic symmetry with lattice constant $d=$ $532 \mathrm{~nm}$. Its depth is determined from Raman-Nath diffrac- 

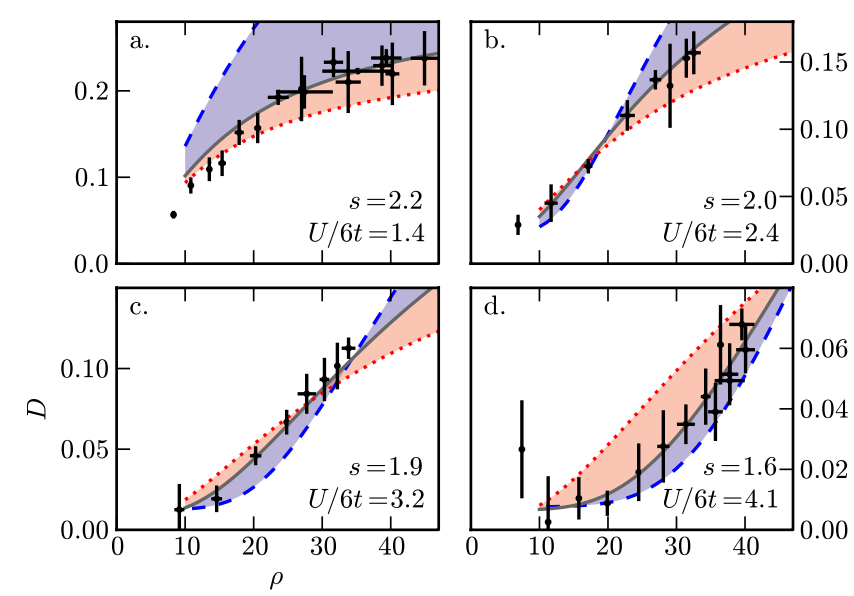

FIG. 1 (color online). Double occupancy: experiment versus theory. Points and error bars are the mean and standard deviation of at least three experimental runs. The solid curve in each panel is the best fit of the second order high-temperature series to the experimental data and yields specific entropies of $s=2.2(2)$, $2.0(5), 1.9(4), 1.6(4)$ for the different interactions strengths of $U / 6 t=1.4(2), 2.4(4), 3.2(5), 4.1(7)$. Curves for $s=1.3$ (dashed curve) and 2.5 (dotted curve) represent the interval of specific entropy measured before and after the ramping of the lattice. We use $k_{B}=1$.

tion of ${ }^{87} \mathrm{Rb}$ and confirmed by resonant excitation of atoms to higher bands [14]. In the lowest Bloch band the tunneling matrix element is $t / h=174(30) \mathrm{Hz}$, where $h$ is Planck's constant. From transverse oscillations in the dipole trap and the standing wave potentials of each lattice beam, we extract overall harmonic trapping frequencies of $\omega_{x, y, z} / 2 \pi=[49.4(9), 52.6(6), 133.0(10)] \mathrm{Hz}$ and a geometric mean of $\omega / 2 \pi=70.1(5) \mathrm{Hz}$. The characteristic filling is $\rho=N / N_{0}$ [15], where $N_{0}=\left(12 t / m \omega^{2} d^{2}\right)^{3 / 2}$ and $m$ is the ${ }^{40} \mathrm{~K}$ atom mass. The characteristic atom number $N_{0}$ corresponds to the atom number per spin that yields half filling in the center of the trap at zero temperature without interaction.

Before loading into the lattice the interaction is tuned by adjusting the scattering length with a Feshbach resonance, whose width was measured using the suppressed dephasing of Bloch oscillations [16] to locate the zero crossing of the scattering length. We obtain a width of 7.5(1) $\mathrm{G}$ which deviates from the result of Ref. [17] where the mean-field energy was measured. We infer the on-site interaction energy $U$ from the scattering length and the Wannier function in the lowest Bloch band [18]. This ab initio $U$ is experimentally validated using resonant excitation of double occupancies by lattice modulation [6,19]. We cover the range from weak repulsion in the metallic regime to strong repulsion with a Mott insulating core using scattering lengths between $200 a_{0}$ and $650 a_{0}$, where $a_{0}$ is the Bohr radius. We choose values of the Hubbard parameter $U / 6 t=1.4(2), 2.4(4), 3.2(5)$, and 4.1(7). Because of the lattice loading process, beam intensity noise, and incoherent photon scattering, the atoms heat up during prepara- tion. Before loading into the lattice, the temperature in the dipole trap is around $0.13 T_{F}$ independent of the atom number as determined from the momentum distribution after time of flight. Here $T_{F}$ is the Fermi temperature. This corresponds to an entropy per atom of $s=S / N \approx$ 1.3 [20]. Since the system is isolated from the environment, the temperature changes significantly even when adiabatically loading into the lattice. The entropy, however, only changes due to nonadiabatic processes. Therefore we can find a typical upper limit of the specific entropy in the lattice by reversing the loading sequence and subsequently measuring the temperature. Here we obtain $s<2.5$.

After loading the atoms into the lattice we determine the double occupancy. A sudden increase of the lattice depth suppresses further tunneling. The fraction of atoms on doubly occupied lattice sites $D$ is then obtained by combining rf spectroscopy, Stern-Gerlach separation of the spin components, and absorption imaging [6,19]. Here we account for the independently determined offset due to the imperfection of the initial spin mixture. From longterm reproducibility and comparison with the adiabatic formation of molecules via magnetic field sweeps, we conclude that the relative systematic uncertainty of the double occupancy measurement is $10 \%$.

Because of the harmonic trapping potential, the temperature behavior of the double occupancy can be markedly different from that of homogeneous systems [11,21]. In a homogeneous system the double occupancy increases with temperature in most regimes of filling and interaction strength due to thermal activation. However, in a harmonically trapped system an increase in temperature allows the atoms to reach outer regions of the trap, in turn reducing the density in the central region: in this case thermal excitations do not populate doubly occupied states but rather deplete them through the decrease of the density. The regimes depicted in Fig. 1 demonstrate the competition between thermal activation and the effect of the trapping potential on the double occupancy as a function of filling and entropy. To extract the entropy the experimental data are compared with theoretical results. The curves in Fig. 1 correspond to the best fitting entropy and its experimental bounds.

We apply a high-temperature series expansion [22] as well as single-site dynamical mean-field theory (DMFT) [23] with a continuous time quantum Monte Carlo solver [24]. In the experimentally relevant regime we find the high-temperature series and DMFT to be in agreement to within $0.2 \%$. For simplicity, the theoretical curves shown in this Letter are therefore generated using the second order high-temperature series unless stated otherwise. The entropy is determined from a one-parameter least-squares fit of the high-temperature series $D\left(s, \rho_{i}\right)$ to the experimental data points $D_{i}$ weighting them according to their statistical errors $\sigma_{D i}$. The fit minimizes $\chi^{2}=\sum_{i}\left(D\left(s, \rho_{i}\right)-\right.$ $\left.D_{i}\right)^{2} / \sigma_{D i}^{2}$. The series is able to accurately reproduce the measured double occupancy for all shown interaction 
strengths. We find deviations of the experimental data only at the lowest fillings at low repulsion, indicating that for very small atom numbers and weak interaction the cooling and loading procedure may fail to produce a constant entropy per atom.

The size and direction of the corridors between the initial and the final entropy in the dipole trap has implications for the usefulness of the double occupancy when performing thermometry. In Fig. 1(a) the behavior for low repulsion $U / 6 t=1.4$ is shown. With increasing filling the system transforms from a dilute gas to an increasingly dense metal with high double occupancy. In this case the effect of the trapping potential dominates and $D$ decreases with increasing entropy. Because of its large $|\partial D / \partial s|$, the regime of Fig. 1(a) is well suited for thermometry.

At intermediate repulsion strengths in Figs. 1(b) and 1 (c), double occupancies become increasingly suppressed and $|\partial D / \partial s|$ decreases. For each interaction strength $\partial D / \partial s$ changes sign at a certain filling. These points mark the crossover to thermal suppression of double occupancies. If $\partial D / \partial s$ vanishes, the theory becomes parameter free to first order and can be used to further determine other calibration factors, e.g., the characteristic filling.

Figure 1(d) shows data for samples in the Mott insulating regime. It exhibits a pronounced plateau of suppressed double occupancy at intermediate fillings owing to a vanishing core compressibility, a characteristic signature of a Mott insulating core [6,12]. Large filling can increase the chemical potential to values comparable with $U$ and thus create double occupancy. In this regime the thermal activation of double occupancies dominates over the thermal decrease of density due to the trapping potential. If $\partial D / \partial s>0$ a large fraction of particles resides in the Mott insulating core. Here the chemical potential is high enough to prevent holes from entering the center and additionally the density of states is sufficiently gapped to allow only few thermally excited double occupancies.

We now consider the systematic errors of all parameters and measurements to assess the absolute reliability of the present method in determining the entropy. Table I lists the contributions. The sensitivity of the least-squares fit to variation of the respective parameter shows the sign of the influence as well as the magnitude. The total relative uncertainties are below $25 \%$ for all four interaction strengths, which confirms the validity of the determined entropies. It is apparent that the systematic errors dominate and that especially the atom number and double occupancy calibrations are critical. The observed increase of the specific entropy with decreasing interaction can be explained by an interaction-dependent global adiabaticity of the preparation [25] or by a combination of systematic errors in $N$ and $D$.

From the theoretical description, several unique properties of trapped repulsively interacting Fermi-Hubbard systems can be derived. Figures 2(a) and 2(b) show the central density $n_{0}$ and compressibility $\kappa_{0}=\partial n_{0} / \partial \mu$ versus characteristic filling for the interaction strengths and specific
TABLE I. Error budget of the entropy determination. The table lists the sensitivity of the fit $\partial s_{\text {fit }} / \partial(\cdot)$ to the changes in the system's parameters scaled by their systematic errors $\delta_{(\cdot)}$. For a positive contribution an increase in the parameter would lead to an increase in the apparent entropy. The contributions are added in quadrature to the fit error estimate $\sigma_{s}^{2}=2\left(\partial^{2} \chi^{2} / \partial s^{2}\right)^{-1}$ to obtain the total uncertainty of the entropy.

\begin{tabular}{lcrrr}
\hline \multicolumn{1}{c}{$U / 6 t$} & 1.4 & \multicolumn{1}{c}{2.4} & \multicolumn{1}{c}{3.2} & \multicolumn{1}{c}{4.1} \\
\hline$\delta_{t} \partial s_{\text {fit }} / \partial t$ & -0.01 & 0.01 & -0.11 & -0.08 \\
$\delta_{U} \partial s_{\text {fit }} / \partial U$ & $\sim 0$ & -0.04 & 0.07 & 0.09 \\
$\delta_{\omega} \partial s_{\text {fit }} / \partial \omega$ & 0.01 & 0.07 & -0.07 & -0.07 \\
$\delta_{N} \partial s_{\text {fit }} / \partial N$ & 0.06 & 0.30 & -0.32 & -0.32 \\
$\delta_{D} \partial s_{\text {fit }} / \partial D$ & -0.16 & -0.30 & 0.13 & 0.13 \\
$\sigma_{s}$ & 0.01 & 0.12 & 0.18 & 0.07 \\
Total $s$ & $2.2(2)$ & $2.0(5)$ & $1.9(4)$ & $1.6(4)$ \\
\hline \hline
\end{tabular}

entropies of Fig. 1. The plateau in $n_{0}$ and the reduction of $\kappa_{0}$ for $U / 6 t=4.1$ are signatures of the Mott insulating regime [21]. Compared to the result for a noninteracting system where the ground state has a compressibility of $1.69 / 6 t$ at half filling, the compressibility is suppressed by a factor of 50 to values as low as $\kappa_{0} \approx 0.03 / 6 t$.

The entropy as determined above needs to be related to a temperature to allow for comparison with models of homogeneous systems. Figure 2(c) shows this temperature in units of the half bandwidth as a function of characteristic filling. The behavior is similar to that of a Fermi gas in a harmonic trap where the temperature at constant specific entropy increases with the atom number [11,20]. At the lowest fillings of $\rho=5$ the temperature in the lattice even
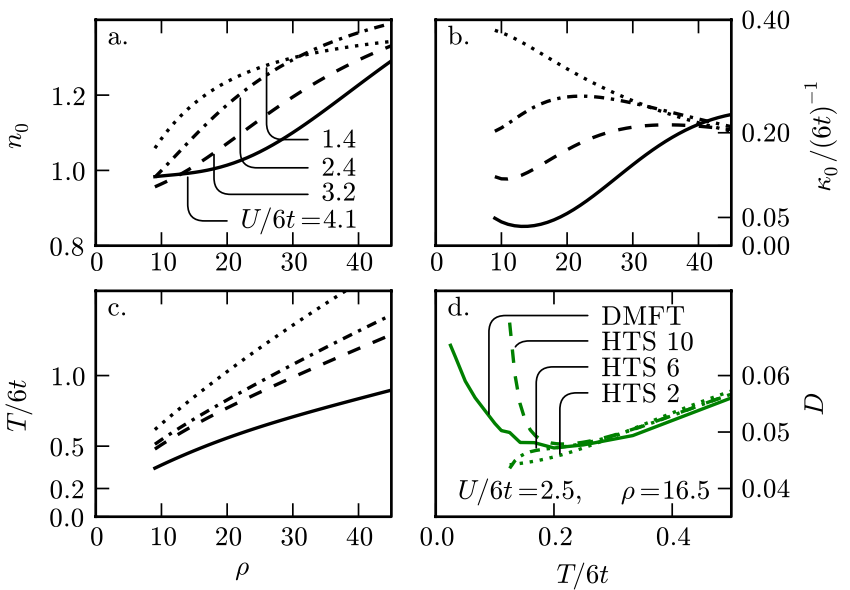

FIG. 2 (color online). Properties of experimental regimes and validity of theoretical methods. Panels (a)-(c) show the central density $n_{0}$, central compressibility $\kappa_{0}$, and temperature $T$ reached in the corresponding Hubbard model as a function of characteristic filling $\rho$ for the parameters of Fig. 1. Panel (d): Agreement between high-temperature series (HTS, second order dotted line, sixth order dash-dotted line, tenth order dashed line) and DMFT (solid line) for $U / 6 t=2.5$ and $\rho=$ 16.5 as a function of temperature in the lattice $T / 6 t$. For low temperatures $T \lesssim t$ the series starts to diverge. 

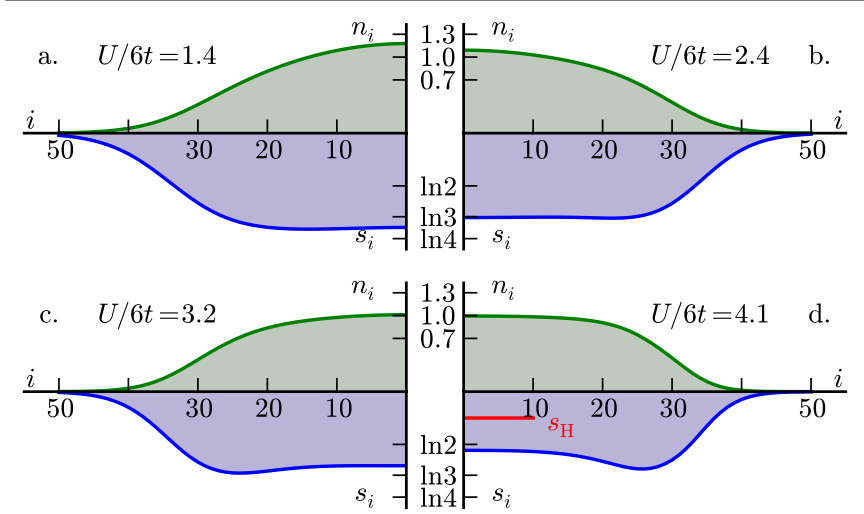

FIG. 3 (color online). Density and entropy distribution in the trap. For the interaction strengths and entropies of Fig. 1 the density $n_{i}$ (upward) and entropy $s_{i}$ (downward) per site $i$ at a characteristic filling of $\rho=15$ in a spherically symmetric system are shown. The buffering effect of the low-density shell around the Mott insulating core becomes clearly visible for $U / 6 t=4.1$. There, the entropy reaches values of about twice the critical entropy of the Heisenberg model $s_{H} \approx \ln 2 / 2$.

approaches the energy scale of the tunneling $T \sim t$. At these low temperatures the results of high-temperature series and DMFT start to deviate considerably; see Fig. 2(d).

In a finite trapped system, antiferromagnetic order requires two conditions to be met. It can only be established in regions of sufficiently constant atom density and low specific entropy. The experimental situation with respect to those conditions is depicted in Fig. 3. The upward axes show the spatial density distribution. At low repulsive interaction [Figs. 3(a) and 3(b)] the system has a density above one in the center which corresponds to a significant doping. In Fig. 3(c), double occupancies and holes compensate in the center and lead to an average density close to unity which then starts to deviate a few sites away from the center. Only for the largest repulsion Fig. 3(d), the Mott insulating core is robust against the confining potential over the central 20 sites. Here, the density changes by less than $1 \%$.

The entropy per site is shown on the downward axes in Fig. 3. It is highest in regions of the trap where the density is most variable. For small interaction strengths it reaches values close to the maximum of $s_{i}=\ln 4$ in some regions. For large repulsion the sites in the perimeter of the cloud where $n_{i} \sim 2 / 3$ carry most of the entropy. This can be understood in the atomic limit at large $U$. At $n_{i}=2 / 3$ each site has three equally likely states and can accommodate $s_{i}=\ln 3$ of entropy. The Mott insulating core can only absorb $\ln 2$ of spin entropy. Mean-field theory of the Heisenberg model predicts this to coincide with the entropy at its critical point. However, quantum fluctuations lower the entropy at the Néel temperature where magnetic long-range order sets in to about $s_{H} \approx \ln 2 / 2 \approx 0.35$ [26].
We have verified numerically that for the Heisenberg model with exchange coupling $J$ the entropy is $s=$ $0.338(5)$ at $T_{\text {Néel }} / J=0.946(1)[27,28]$ by integrating the energy, $S=\int d E / T$. Integration from above or below the Néel temperature agree. Additionally, we performed a new study of the Hubbard model using a diagrammatic determinant Monte Carlo method [29]. For $U / t=8$, the critical temperature is $T_{\text {Néel }} / t=0.325(7)$ [30], and the critical entropy $s_{\text {Néel }}=0.345(45)$. This differs from the mean-field calculation including fluctuation corrections of Ref. [31] and is a factor of 2 less than the experimental results presented here.

We acknowledge funding from SNF, NCCR MaNEP, NAME-QUAM (EU, FET-Open), SCALA (EU), ANR (FABIOLA and FAMOUS), DARPA-OLE, and "Triangle de la Physique."

*esslinger@phys.ethz.ch

[1] B. DeMarco and D. S. Jin, Science 285, 1703 (1999).

[2] T. Loftus et al., Phys. Rev. Lett. 88, 173201 (2002).

[3] M. Greiner et al., Nature (London) 415, 39 (2002).

[4] M. Köhl et al., Phys. Rev. Lett. 94, 080403 (2005).

[5] W. Hofstetter et al., Phys. Rev. Lett. 89, 220407 (2002).

[6] R. Jördens et al., Nature (London) 455, 204 (2008).

[7] U. Schneider et al., Science 322, 1520 (2008).

[8] S. Trotzky et al., arXiv:0905.4882v1.

[9] D. Weld et al., Phys. Rev. Lett. 103, 245301 (2009).

[10] N. Strohmaier et al., Phys. Rev. Lett. 99, 220601 (2007).

[11] M. Köhl, Phys. Rev. A 73, 031601 (2006).

[12] V. W. Scarola et al., Phys. Rev. Lett. 102, 135302 (2009).

[13] G. Reinaudi et al., Opt. Lett. 32, 3143 (2007).

[14] O. Morsch and M. Oberthaler, Rev. Mod. Phys. 78, 179 (2006).

[15] M. Rigol et al., Phys. Rev. Lett. 91, 130403 (2003).

[16] M. Gustavsson et al., Phys. Rev. Lett. 100, 080404 (2008).

[17] C. A. Regal and D.S. Jin, Phys. Rev. Lett. 90, 230404 (2003).

[18] D. Jaksch et al., Phys. Rev. Lett. 81, 3108 (1998).

[19] N. Strohmaier et al., Phys. Rev. Lett. 104, 080401 (2010).

[20] L. D. Carr et al., Phys. Rev. Lett. 92, 150404 (2004).

[21] L. De Leo et al., Phys. Rev. Lett. 101, 210403 (2008).

[22] J. Oitmaa et al., Series Expansion Methods for Strongly Interacting Lattice Models (Cambridge University Press, Cambridge, England, 2006).

[23] A. Georges et al., Rev. Mod. Phys. 68, 13 (1996).

[24] P. Werner et al., Phys. Rev. Lett. 97, 076405 (2006).

[25] C.-L. Hung et al., arXiv:1003.0855v1.

[26] F. Werner et al., Phys. Rev. Lett. 95, 056401 (2005).

[27] A. W. Sandvik, Phys. Rev. Lett. 80, 5196 (1998).

[28] S. Wessel, Phys. Rev. B 81, 052405 (2010).

[29] E. Burovski et al., Phys. Rev. Lett. 101, 090402 (2008).

[30] This estimate improves on the earlier result $T_{\text {Néel }} / t=$ 0.31(1) of R. Staudt et al., Eur. Phys. J. B 17, 411 (2000).

[31] A. Koetsier et al., Phys. Rev. A 77, 023623 (2008). 